\title{
Using Smart Phones and Body Sensors to Deliver Pervasive Mobile Personal Healthcare
}

\author{
Patrick Crilly ${ }^{1}$, Vallipuram Muthukkumarasamy ${ }^{2}$ \\ School of Information and Communication Technology, \\ Griffith University, Gold Coast, Australia \\ ${ }^{1}$ patrick.crilly@griffithuni.edu.au \\ ${ }^{2}$ v.muthuegriffith.edu.au
}

\begin{abstract}
Pervasive health care is regarded as a key driver in reducing expenditure and enabling improvements in disease management. Advances in wireless communication and sensor technologies permit the real time acquisition, transmission and processing of critical medical information. In this paper, we examine different approaches of streaming physiological data from body sensors over a wireless network. Modern mobile phones provide sufficient storage and computational abilities and provide a flexible programming environment, making them ideal to process and store sensed data from multiple sources. We compare the approach of using a central data server, against using a smart phone, to store and process the medical data. The competing requirements of minimization of energy consumption versus the timely delivery of anomalous conditions are investigated using a simulated body sensor network. The measurements show that when a patient is mobile, a smart phone is the device best suited to perform the initial processing of vital signs and sending of medical alerts.
\end{abstract}

\section{INTRODUCTION}

In Western countries, the aging population and the rise of chronic diseases are placing increasing pressure on the cost of providing health care. For example, Australian Government expenditure on health and aged care is expected to rise from $9 \%$ to $12.4 \%$ of GDP (i.e. $\$ 246$ billion) by 2020 [1]. Furthermore it is estimated that 30 to 50 per cent of patients with chronic disease are hospitalised because of inadequate care or management [2]. The global shortage of qualified health care professionals is also contributing to a higher burden on those in the industry [3]. Technology has often provided a platform for enhancing the provision of quality healthcare and for driving down the health expenditure.

A wireless sensor network consists of a large number of distributed nodes. The nodes are designed to be small, cheap and autonomous. Each node consists of a microcontroller, a transceiver, an energy source and a sensor. The nodes are usually connected in an ad-hoc arrangement. Wireless sensor networks are deployed in a variety of application areas, such as environment and habitat monitoring, healthcare and traffic control.

Body area networks can be considered as a special type of wireless sensor networks with their own specific requirements. They differ in nature and requirements from traditional widearea wireless sensor networks, with the most important ones being the increased demand for reliability, energy efficiency and mobility support. The sensor nodes are designed to be worn on the body or implantable. IEEE has identified three application domains where body sensor networks are useful; disease management, health and fitness, and independent living [4]. In the later case, a home health care network, comprising body sensors, cameras and a home health controller, can be used to monitor a patient's well being in their home environment [5].

The home health controller is expected to reside permanently within the home, so when a patient leaves their residence, another device must act as the control node for the body sensor network. The mobile phone has already been proposed to act as a control node [6]. It provides a far more sophisticated human interface than the limited interfaces found on a sensor node. Mobile phones are equipped with several services capable of communicating with a centralised server e.g. SMS, MMS, and GPRS. Modern phones, such as the iPhone, are equipped with cameras, accelerometers and GPS devices, which can be utilised to provide contextual data about a patient. The phone itself can be used to enter other information or to record a patient's emotional or mental state. As demonstrated by Liu et al. [7], this feature can be used to enhance a patient's compliance to medical program or adherence to a prescribed drug regimen. Typically Bluetooth has been used as the wireless technology to interconnect the mobile phone and the sensors, as it generally found on commercially available phones. In general, the mobile phone penetration is very high both in developed and developing countries. For example, there are more than 22 million phones owned by Australians and about $98 \%$ of the population has access to a mobile phone network.

In this research, we examine the feasibility of using the mobile phone as a control node to provide mobility and to perform analysis and diagnosis of medical conditions, when acting as the control node in a personal healthcare system. Section II outlines some of the relevant research projects into body sensor networks. The ability of various devices in a body sensor to perform processing of physiological parameters is examined. Section III proposes architecture for a personal healthcare system and identifies a number of requirements the system should fulfil. Section IV details a testbed for measuring some metrics for the proposed system. Section V presents and discussed the results of these measurements. Finally conclusions are reported in Section VI. 
TABle I: SAMPle IMPLEMENTATIONS OF USING A MOBILE DEVICE FOR MONITORING

\begin{tabular}{|l|l|l|l|}
\hline Platform & Base Device & Wireless Protocol & Function \\
\hline HealthGear & Phone & Bluetooth & Wearable system for connecting sensors and mobile phones \\
\hline CodeBlue & PC, PDA & 802.15 .4 & Provision of medical monitoring in a hospital environment \\
\hline ALARM-NET & $\begin{array}{l}\text { PC, PDA, } \\
\text { Stargate }\end{array}$ & Bluetooth 802.11 & $\begin{array}{l}\text { Wireless sensor network for } \\
\text { assisted-living and residential monitoring. }\end{array}$ \\
\hline DexterNet & $\begin{array}{l}\text { PC, PDA, } \\
\text { Phone }\end{array}$ & 802.15 .4 & Body sensor network for indoor and outdoor monitoring. \\
\hline
\end{tabular}

\section{RELATED WORK}

A range of software and hardware architectures have been proposed and implemented to evaluate and address the needs of body sensor networks in health care applications. Table I summarises some of the major research projects into body sensors networks and monitoring [8-11].

The HealthGear project implemented a wearable real-time monitoring solution for the detection of apnea events. A pulse oximeter was connected to a mobile phone using the Bluetooth protocol. The project demonstrated the feasibility of storing and processing of physiological data on a mobile phone. The Harvard University research project, CodeBlue, developed a number of wireless sensors for monitoring vital signs and examined the benefits to clinicians, nurses, emergency personal in hospital environments and disaster situations, of connecting the sensors in an ad-hoc arrangement and relaying the data. The Alarm-Net project developed a system architecture for assisted living and residential monitoring. The Alarm-Net architecture has been used by several other projects to investigate issues surrounding wireless body sensors and healthcare. DexterNet is open source platform that uses a three layer architecture to support monitoring in indoor and outdoor environments. The layers are the Body Sensing Layer (BSL) which is used to interconnect sensing for monitoring the biological signals; the Personal Network Layer (PSL), which co-ordinates the BSL; and the Global Network Layer (GNL), which supports high level applications such as remote health monitoring.

To evaluate where storage and analysis of data should take place in a body sensor network, we categorise them into three distinct approaches to data processing and detection of abnormal conditions:

1. Continuously streaming data from the sensors to a control node. The control node then acts as an aggregator of the data, forwarding it on to a remote server where diagnosis is performed.

2. The sensors are programmed to detect and raise alerts when abnormal conditions are detected. These alerts are sent to the control node which forwards them on to an emergency centre or medical practitioner, so appropriate action can be undertaken.

3. Data is continuously streamed to a control node, where the analysis is performed. The control node processes the data and raises alerts when abnormal conditions are detected. These alerts are then sent to emergency centre etc.

We examine the research utilising body sensor networks to aid in the detection of cardiac arrhythmias to explore the relative merits of each approach. Cardiac arrhythmia is an irregular heart beat or abnormal heart rhythm. Most arrhythmias are harmless, but some can indicate a serious or life threatening condition. Some arrhythmias occur infrequently, making them hard to diagnose. An ECG, which records the electrical activity of the heart is the most method used to diagnose arrhythmias.

Yousef \& Lars [12] demonstrated that an ECG sensor and mobile phone could be integrated into a usable system for remote cardiac monitoring. A remote server was used to analyse the data. Their system had an uptime of $95.5 \%$ and $90 \%$ of ECGs were delivered reliably. As transmitting data over the cellular network is a large drain on a phone's battery, the reliability was achieved by powering the phone from a separate battery package and having the heart patient carry an extra mobile phone. Continuous streaming of data is therefore unsustainable in terms of a phone's battery life.

Wireless data transmission is the major source of energy consumption from the sensor. The approach adopted by Rincon, et al. [13] was to implement ECG analysis in a sensor. They showed that energy consumption of the sensor could be reduced by $92.6 \%$ by detecting and sending pertinent points of the ECG signal. They also showed that if complete diagnosis is performed on the sensor, the reduction in transmission is $100 \%$ if no abnormalities are detected. The limitations of this approach are that there is no data saved for verification or auditing of the analysis and that the algorithm used for detection is not easily updated.

Oresko, et al. [14] implemented a prototype system, where ECG analysis and classification is performed by streaming data from an ECG heart monitor to a smart phone. This approach represents a compromise between conserving power in the system and permitting data analysis and storage. 


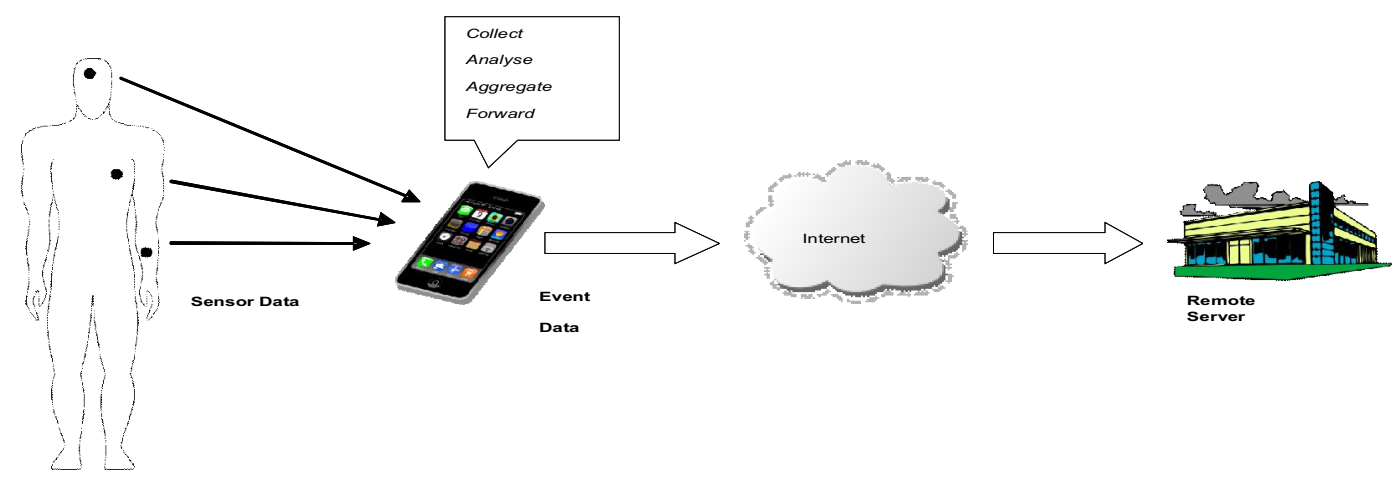

Figure 1: Proposed personal flexible health system for capturing vital signs

\section{Proposed Personal HealthCare System}

In this section, we examine and identify the requirements in terms of communication, storage, processing that need to be satisfied for an acceptable healthcare system. The most appropriate architecture is investigated and proposed.

The control node in the personal health system should be able to perform the following functions

- Be able to analyse and process vital signs for abnormal conditions using limits and techniques that are adapted to a patient's personal history.

- $\quad$ Be able to store data for remote queries, auditing and comprehensive analysis.

- Be flexible. The system should have the ability to have algorithms downloaded as required and permit the detection metrics to be updated remotely.

- Be able to send alarms and associated vital signs in timely manner, when medical alerts are detected. These vital signs can undergo further analysis on a remote expert system or by a clinician.

Figure 2 presents a proposed architecture for personal health system that can be used for monitoring patient's vital signs. The system is used to add mobility to a home health care server, so that a patient need not be confined to their place of residence but instead be able to participate in their usual day-to-day activities.

The personal health system depicted consists of the patient with a number of implanted or attached body sensors, smart phone with Internet connectivity to a remote server

1. A set of wireless sensors for measuring the vital signs of blood pressure, heart rate, temperature and respiration rate are either implanted or attached to the body. The acceptable ranges of the physiological values vary depending on the activity level of the patient, so an accelerometer is included in this instance to provide some basic context information about the activity level of the patient e.g. have they had a fall, are they running or at rest.
2. A smart phone which acts as the control node in the network. There is no clear industry definition of what constitutes a smart phone. We use the term smart phone to distinguish between a phone which offers basic features such a making calls and a phone which offers a richer set of built-in applications and provides Internet connectivity, the later being defined as a smart phone. Smart phones are distinguished by a more powerful processor, larger screen and more memory than that of a basic mobile phone. With Bluetooth being widely available on mobile phones, it is adopted as the lowpower communication protocol for connecting the sensors to the mobile phone. The phone is responsible for discovering and configuring the connections to the sensors.

The sensors are arranged in a star network, since they are heterogeneous in nature and do not exchange information between each other.

3. A server, which may be a doctor's office, hospital or a remote monitoring service. Abnormal events and alarms are sent to the server when they are detected. Clinical data pertaining to the event can either be retrieved from the phone or sent to the server over the Internet using a $3 \mathrm{G}$ mobile telephony protocol.

As a patient leaves their residence, the control node performs network assembly. The sensors remain connected to the mobile phone and transmitting physiological data for the duration of the patient's absence from their home. To preserve energy whilst not communicating, one of the Bluetooth low power states can be utilised. While the patient is mobile, the phone is responsible for analysing the vital signs, storing them to the phone, and detecting and sending alerts when abnormal conditions are detected.

Communicating with the public interface from the phone is expensive in terms of the energy and the network connection is unreliable. Furthermore, receiving the raw data in real-time is not the primary function of the system. The focus is detecting when parameters exceed predefined bounds or anomalies are detected. When an anomaly is detected, a doctor or clinician can query the phone and request the phone 
to send the data pertaining to this event. The delay between requesting and receiving this window of information is important, since time to respond to a medical emergency and in many other situations can be critical in determining the outcome.

Analysing and storing sensor data and on the phone represents a trade off between conserving energy and timely detection and reporting of abnormal conditions. Section IV discusses a test setup and measurements used to analyse this trade off.

\section{EXPERIMENTS}

To assess the relative merits of continuously streaming data to a remote sever against analysing and storing data on the mobile phone, we examined two test scenarios:

1. An ECG sensor is used to continuously stream samples to a remote server via a mobile phone connected to the Internet.

2. Samples are sent from an ECG sensor to a mobile phone where they are stored. After a number a samples are sent an alert is simulated by sending the stored samples to a remote server.

We are not concerned with the actual analysis of the sensed data. It is assumed that same algorithm for processing of the data or appropriately modified version can be implemented equivalently on either the remote server or on the mobile phone, and the difference in the compute times between the two platforms is negligible relative to packet transmission times and other delays.

\section{A. Testbed}

The testbed show in the figure below is used to perform the measurements. The testbed consists of a simulated wireless ECG sensor connected to mobile phone via Bluetooth. A PC which is connected to the Internet acts as the remote server

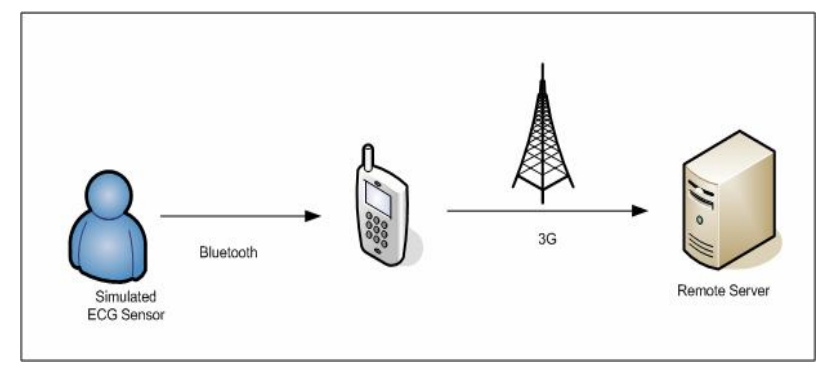

Figure 2: Test Setup: simulated ECG sensor, mobile phone and remote server

This setup is used to measure the time taken to transmit the data and the power consumption in both the scenarios. For the first case - continuously streaming samples, time taken to send the sensed data from the sensors to the mobile phone and then from the phone to the server are recorded. For the second case, the time taken to connect and transmit stored data from the phone to the server is measured. Power consumption by the mobile phone is also measured.

\section{1) Wireless ECG Sensor}

To simulate a wireless ECG sensor, a $\mathrm{C}$ application is developed and used. The MIT-BIH Arrhythmia Database is utilised to provide ECG data. The database contains 48 halfhour excerpts of two-channel ambulatory ECG recordings, obtained from 47 subjects [16].

The application reads samples using a single record from the database. To permit timing calculations to be performed, the application sends a packet containing the current time and the sampled value every $2.7 \mathrm{~ms}$, as the ECG recordings were digitized at $360 \mathrm{~Hz}$.

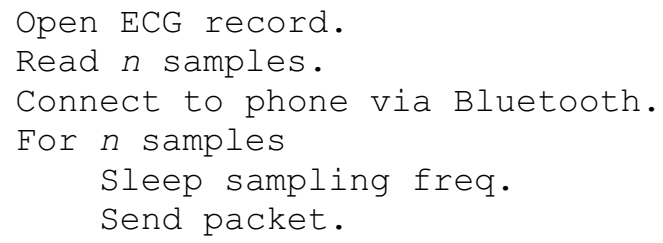

Figure 3: Pseudo code for simulated ECG sensor

\section{2) Mobile Phone}

The timing measurements were performed using a Nokia E65 mobile phone. To perform power consumption measurements, a Nokia N95 mobile phone running the Nokia Energy Profiler MIDlet was used. Internet connectivity was established using a commercial $3 \mathrm{G}$ network offering 7.2 Mbps HSDPA and 2 Mbps HUSPA services.

For each of the test scenarios a MIDlet written in J2ME is used on the phone. The pseudo code for the MIDlets used to implement the test scenarios of continuous streaming of sensor data and storing data to the phone is shown in figure and figure below.

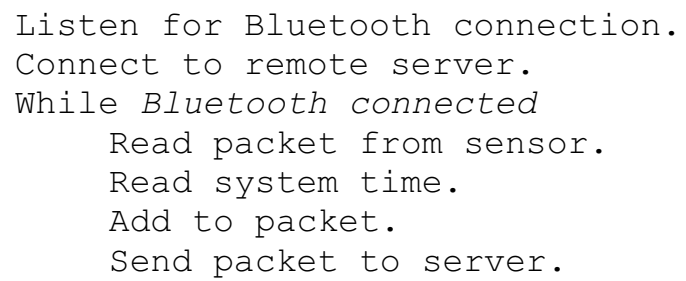

Figure 4: Pseudo code for MIDlet which handles continuos streaming of sensor data.

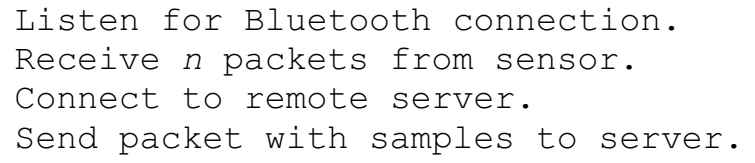

Figure 5: Pseudo code for MIDlet which stores sensor data and sends an alert

\section{3) Server}

A C application on the server listens for incoming connections from the Internet. For the data streaming test case, it reads a fixed number of packets and performs timing calculations on them. In the second scenario, the application waits for the connection from the mobile phone simulating an alert. The application then receives the packet containing the alert data and calculates the time it took to send. 


\section{4) Synchronising Time}

The client program which simulates the wireless ECG sensor and the server application are run the same $\mathrm{PC}$ to ensure that the timing calculations are accurate. The PC also runs an NTP server daemon. Before each test, the FreeTimeBox [17] application is run on the mobile phone to synchronise time with the PC.

At the start of each set of timing measurements, the time delta between the phone and the server is determined by sending 20 packets from the server to the mobile phone, and have the packets echoed back with the time on the phone included in them.

\section{Results AND Discussion}

In this section we summarise and discuss the results obtained from our experiments using the testbed in Figure 2 and the two test scenarios outlined in section IV.

\section{A. Comparison of Energy Consumption.}

Figure 6(a) and 6(b) show the power consumption graphs obtained when continuously streaming data and when storing the same samples on the phone respectively.

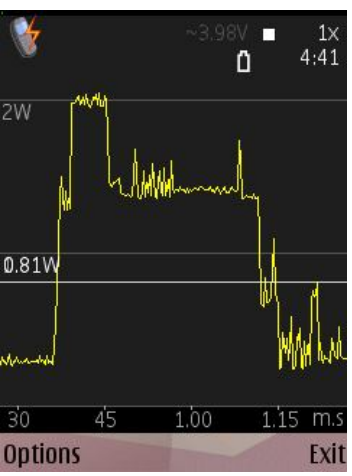

(a)

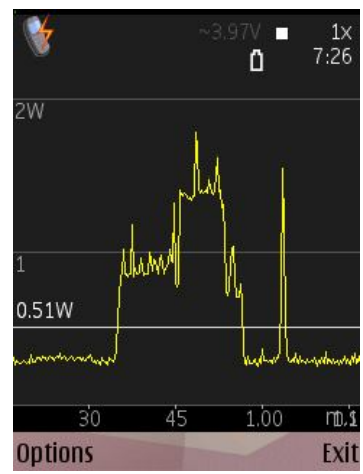

(b)
Figure 6: Power consumption graphs

Figure 6(a) shows that power consumption is about 0.3 Watts while waiting for a Bluetooth connection. This rises to 2 Watts as the sensor connects and starts streaming samples. About 1.4 Watts is consumed for continuous streaming of sensor data. The value in top right corner (4:41 hours) shows the life of a fully charged battery at the average power consumption, which in this case is 0.81 Watts.

Figure 6(b) shows power consumption of about 0.9 Watts for receiving streaming samples over Bluetooth and rising to about 1.3 Watts when sending the stored data over the $3 \mathrm{G}$ network to remote server. The average power consumption shows that battery would last 7.26 hours. Considering the maximum amount of time permit a home-monitored may need to be away from their residence, this value seems reasonably adequate.

The results of the power consumption measurements are summarised in Table II. By storing samples on the phone around $39 \%$ energy saving is achieved, thus obtaining an almost $65 \%$ increase in battery lifetime.
TABLE II: SUMMARY OF POWER CONSUMPTION MEASUREMENTS

\begin{tabular}{|l|l|}
\hline Action & Average Power (W) \\
\hline Listening for Bluetooth connections & 0.30 \\
\hline Sending samples over Bluetooth & 0.9 \\
\hline Continuously streaming samples & 1.4 \\
\hline Sending alert data to remote server & 1.3 \\
\hline
\end{tabular}

\section{B. Continuous Streaming of Sensor Data}

Table III below shows the results obtained when continuously streaming data from the wireless ECG sensor to the remote server via the mobile phone. Time taken to send the data to the server vary widely as indicated by the standard deviation. Although it is not very clear why this is the case, we assume that it could be due to the fact that some other activity is simultaneously taking place on the phone which affects transmission over the $3 \mathrm{G}$ network.

TABLE III: TIMES TO SEND ECG SAMPLES WHEN STREAMING

\begin{tabular}{|l|r|r|r|r|}
\hline & min (ms) & max (ms) & avg (ms) & stdv (ms) \\
\hline Bluetooth & 50 & 122 & 62.59 & 9.31 \\
\hline 3G & 92 & 1485 & 343.76 & 314.87 \\
\hline Total & 145 & 1543 & 406.35 & 316.29 \\
\hline
\end{tabular}

\section{Storing Samples on the Phone}

Table IV below shows the time it takes to connect and forward 300 and 600 data samples to the server respectively. As the sampling rate of ECG data was 360 samples per second, the values of 300 and 600 were chosen to approximate one and two heart beats. In the absence of a heart rate calculated from the sensor data, this represents a patient with a heart rate of 72 beats per minute, inside the normal pulse rate range of 60 to 80 beats per minute for a healthy adult.

TABLE IV: RESPONSE TIMES TO AN ANOMALY

\begin{tabular}{|l|c|c|}
\hline \# of samples stored & 300 & 600 \\
\hline Response time & $10686 \mathrm{~ms}$ & $19727 \mathrm{~ms}$ \\
\hline
\end{tabular}

Although the response time is $10-20$ seconds, depending on the sample data size, given the savings in energy consumption, the increased response times to abnormal conditions, justify storing sensed data on the phone rather than continuously sending the samples to a remote server.

\section{Flexibility}

Programming the phone did not require any special tools and updates to the MIDlet were easily applied by connecting the phone via USB to a PC. This demonstrates the flexibility of adopting the mobile phone as the platform for acting as the control node in a body sensor network.

The sensors are by their nature designed as an embedded systems and therefore don't provide a framework for updating by the user. Smart phones easily support installation and update of applications. Vendors such as Apple, Nokia and Google already provide infrastructure via the AppStore, Ovi and AppsMarketPlace respectively to download and install applications for their products. Therefore new or additional 
programs or algorithms for detecting medical conditions could be relatively easily installed onto the phone in response to specific events being detected or as the medical needs dictate.

\section{CONCLUSIONS}

To provide mobility in a personal health system for monitoring vital signs, the mobile phone is a convenient and suitable device to act as the control node. The phone has become the device ubiquitous for personal communication and data access. It is rich in functionality and can be utilised to provide additional sensed data, either from on board sensors or from user input. Furthermore, the new technological trends towards developing networks to satisfy human-to-human $(\mathrm{H} 2 \mathrm{H})$ and machine-to-machine (M2M) communication will only enhance the suitability of the use of phone in the pervasive healthcare system.

In this paper, we have shown that by storing samples on the mobile phone instead of continuously streaming data to a remote server results in a significant saving in battery life with little impact on the timely delivery of abnormal events and their associated data.

No consideration has been given to the utility nature of the device. Besides making calls, mobile phones are also used for taking photos, playing MP3s, playing games, etc. Further research should examine the impact these activities may have on the performance of the device when acting as a control node in a body sensor network.

\section{REFERENCES}

[1] Minister for Health and Aging, The Honorable Nicola Roxon MP, "Speech Opening Address," in Health e-Nation Conference, National Convention Centre, Canberra, 2009.

[2] B. K. Armstrong, et al., "Challenges in health and health care for Australia," Med J Aust, vol. 187, pp. 485-9, Nov 52007.

[3] T. Evans, editor, The World Health Report 2006. Working together for health: World Health Organization, 2006.

[4] R. Carroll, et al., "Continua: An interoperable personal healthcare ecosystem," Pervasive Computing, IEEE, vol. 6, pp. 90-94, 2007.

[5] K. Singh and V. Muthukkumarasamy, "Authenticated key establishment protocols for a home health care system ", Proceedings of the Third International Conference on Intelligent Sensors, Sensor Networks and Information, Melbourne, Australia, 2007, pp. 353-358.

[6] F. Fitzek and S. Rein, "Convergence of Mobile Devices and Wireless Sensor Networks," ed, 2007, pp. 375-380.

[7] W. T. Liu, et al., "Efficacy of a cell phone-based exercise programme for COPD," Eur Respir J, vol. 32, pp. 651-9, Sep 2008.

[8] P. Kuryloski, et al., "DexterNet: An open platform for heterogeneous body sensor networks and its applications", Sixth International Workshop on Wearable and Implantable Body Sensor Networks, 2009, pp. 92-97.

[9] D. Malan, et al., "CodeBlue: An ad hoc sensor network infrastructure for emergency medical care," Workshop on Wearable and Implantable Body Sensor Networks, 2004.

[10] N. Oliver and F. Flores-Mangas, "HealthGear: A real-time wearable system for monitoring and analyzing physiological signals," in Wearable and Implantable Body Sensor Networks, 2006. BSN 2006. International Workshop on, 2006, pp. 4 pp.-64.

[11] A. Wood, et al., "ALARM-NET: Wireless sensor networks for assisted-living and residential monitoring," Department of Computer Science, University of Virginia, 2006.

[12] J. Yousef and A. N. Lars, "Validation of a real-time wireless telemedicine system, using bluetooth protocol and a mobile phone, for remote monitoring patient in medical practice," Eur J Med Res, vol. 10, pp. 254-62, Jun 222005.
[13] F. J. Rincon, et al., "Implementation of an automated ECG-based diagnosis for a wireless body sensor platform," presented at the International Conference on Biomedical Electronics and Devices (BIODEVICES 2009), Porto, Portugal, 2009.

[14] J. J. Oresko, et al., "A wearable smartphone-based platform for realtime cardiovascular disease detection via electrocardiogram processing," IEEE Transactions on Information Technology in Biomedicine, vol. 14, pp. 734-740, 2010.

[15] W. R. Mower, et al., "Pulse oximetry as a fifth pediatric vital sign," Pediatrics, vol. v99, p. p681(6), 1997.

[16] A. L. Goldberger, et al., "PhysioBank, PhysioToolkit, and PhysioNet : Components of a new research resource for complex physiologic signals," Circulation, vol. 101, pp. 215-220, June 13, 2000.

[17] FreeTimeBox. (2010, 30 August). [Online]. Available: http://handheld.softpedia.com/get/Business/Clock/FreeTimeBox64856.shtml 